\title{
LATERAL VEHICLE STABILIZATION USING CONSTRAINED NONLINEAR CONTROL
}

\author{
Petter Tøndel*, Tor A. Johansen* \\ * Department of Engineering Cybernetics,Norwegian University of Science and Technology, \\ N-7491 Trondheim, Norway, \{Petter.Tondel, Tor.Arne.Johansen\}@itk.ntnu.no
}

Keywords: Automotive control, optimal control, nonlinear systems, constraints.

\begin{abstract}
A lateral stabilization system for automotive vehicles is designed based on nonlinear constrained receding horizon control. An explicit piecewise linear control law approximating the optimal solution is computed off-line, giving a computationally efficient on-line solution which can be implemented with low real-time software complexity on inexpensive hardware. Simulations show that the controller is able to stabilize the vehicle in extreme maneuvers where the vehicle otherwise becomes unstable.
\end{abstract}

\section{Introduction}

One of the first contributions within the field of stabilizing control of automotive vehicles was ABS brakes, which was commercially introduced more than 20 years ago. ABS controllers maintain high longitudinal braking efficiency and improve lateral stability of the vehicle during braking maneuvers. These systems were followed by traction control systems, which improve the lateral stability and maximize friction during acceleration of the vehicle. A natural continuation of this development was to introduce automatic control of the yaw motion during critical situations to retain steerability of the vehicle, see e.g. [1]. Such control systems became commercially available in the 90's, with the Electronic Stability Program (ESP) [2].

The contribution of this work is a study of model-based, constrained optimal control of the vehicle yaw dynamics. As suggested in [10] the strategy is to keep the states within certain bounds so that steerability of the vehicle is retained. This is done by using a receding horizon control (RHC) approach in which a nonlinear optimization problem is solved at each sample, taking advantage of all available degrees of freedom in the braking system. We are not aware of any previous work on optimization based controllers for automotive vehicle yaw dynamics.

The main problems of designing a controller for the yaw dynamics are the nonlinearities which appear in the system model, in addition to achieving the largest possible region of stabilizability under control input constraints. The RHC problem formulated in this paper requires the solution of a nonlinear program (NLP) at each control sample, giving an optimal feedback control. This is, however, not a technique which is applicable to a practical implementation in a real vehicle, due to high on-line computational requirements, and complex numerical software which is generally not recommended in safetycritical applications. We address this problem by designing an approximation to the optimal solution based on multiparametric nonlinear programming (mpNLP) [8]. This is a technique in which an arbitrarily close approximation to the solution of an NLP can be found off-line as an explicit, piecewise linear (PWL) function of the state. This gives a solution which can readily be implemented and verified on inexpensive hardware with high software reliability.

\section{Vehicle Model}

In the model-based approach to yaw rate control we use the vehicle model for horizontal plane motion in [11], where more details can be found.

Some nomenclature:

$v \quad$ Speed (absolute value of velocity vector at COG)

$\beta \quad$ Vehicle side slip angle

$\dot{\psi} \quad$ Yaw rate

$F_{x i} \quad$ Force on wheel in longitudinal direction, $i \in\{1,2,3,4\}$

$F_{y i} \quad$ Force on wheel in lateral direction, $i \in\{1,2,3,4\}$

$F_{z i} \quad$ Vertical force on ground from each wheel, $i \in\{1,2,3,4\}$

$\delta_{i} \quad$ Steering angle, $i \in\{1,2,3,4\}$

$m \quad$ Vehicle mass

$J \quad$ Vehicle moment of inertia about COG

$\mu_{H} \quad$ Maximum friction coefficient

$\alpha_{i} \quad$ Wheel side slip angle (angle between velocity vector at centre of wheel and wheel direction), $i \in\{1,2,3,4\}$

$\mu_{y i} \quad$ Lateral friction coefficient, $i \in\{1,2,3,4\}$

$\mu_{x i} \quad$ Longitudinal friction coefficient, $i \in\{1,2,3,4\}$

A three state model will be used to describe the dynamics of the vehicle, based on the geometry in Figure 1. We will assume that the driver controls the front wheel steering angle $\delta_{W}$ by using the steering wheel, while the controller can use the four longitudinal brake slips $\lambda_{x i}$ for stabilizing the lateral motion. We assume that only brakes are available as actuators, so that only negative forces $F_{x i}$ can be generated. Moreover, we assume that only the front wheels can be steered, that is $\delta_{1}=\delta_{2}=\delta_{W}$, and $\delta_{3}=\delta_{4}=0$. The wheel forces are given by the friction coefficients, which are given by nonlinear functions (friction curves) [11]:

$$
\begin{aligned}
& F_{x i}=-F_{z i} \mu_{x i}\left(\lambda_{x i}, \alpha_{i}, \mu_{H}\right) \\
& F_{y i}=F_{z i} \mu_{y i}\left(\lambda_{x i}, \alpha_{i}, \mu_{H}\right) .
\end{aligned}
$$

For simplicity we have assumed $F_{z i}=\frac{m g}{4}$. One could, however, include the effects of roll and pitch on $F_{z i}$ without adding 


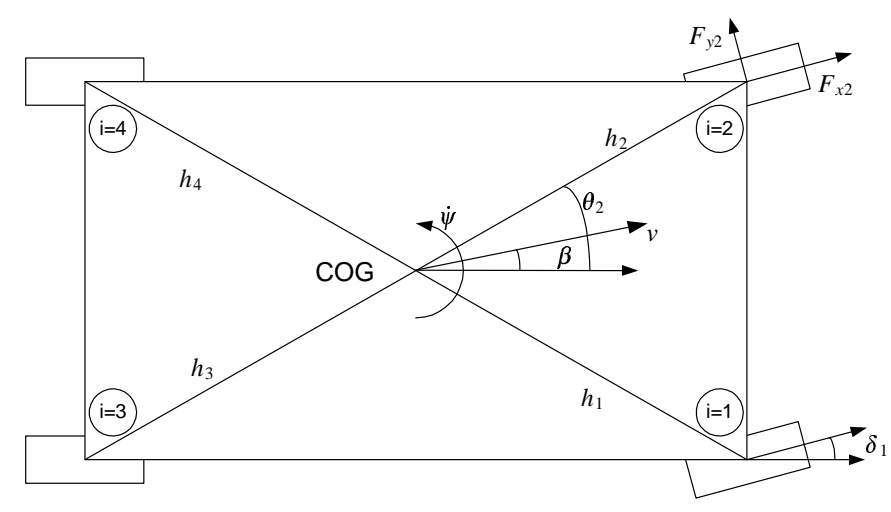

Figure 1: Vehicle geometry and coordinate definitions

considerably to the complexity of the control law. Figure 2 shows friction curves for lateral forces for some values of $\alpha_{i}$, while Figure 3 shows a typical friction curve for longitudinal forces for $\alpha_{i}=0$. The vehicle is assumed to be equipped with an ABS system with a slip controller which can apply commanded longitudinal slip values individually to each wheel, $[5,11,13]$.

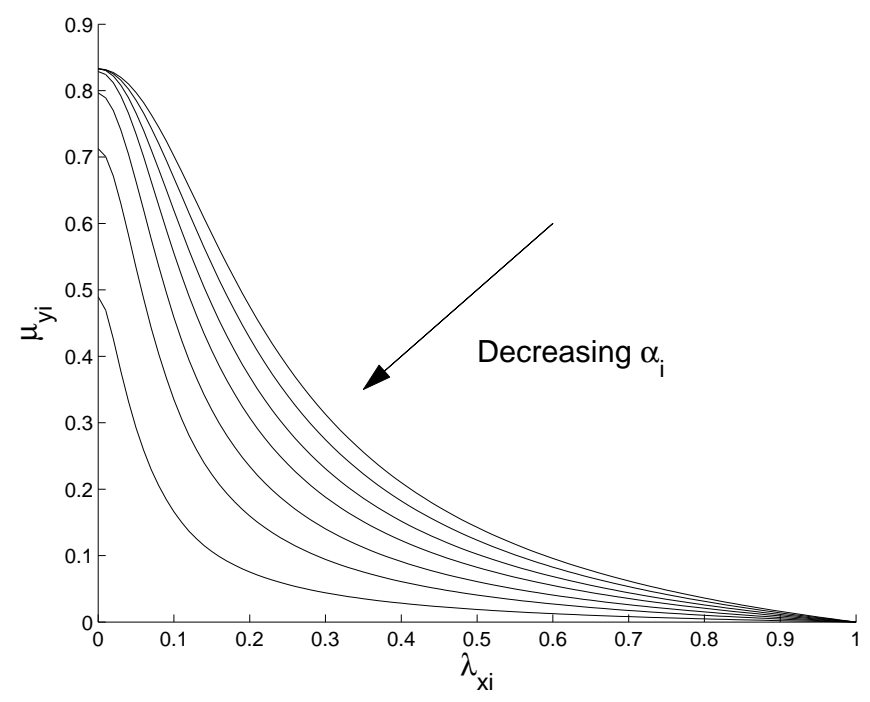

Figure 2: Lateral friction coefficient as a function of the longitudinal slip

From Newton's law one can derive

$$
\left[\begin{array}{c}
\dot{v} \\
\dot{\beta}
\end{array}\right]=-\left[\begin{array}{c}
0 \\
\dot{\psi}
\end{array}\right]+\frac{T(v, \beta)}{m} \sum_{i=1}^{4} D\left(\delta_{i}\right)\left[\begin{array}{c}
F_{x i} \\
F_{y i}
\end{array}\right] .
$$

Similarly, a torque balance gives

$$
\ddot{\psi}=\frac{1}{J} \sum_{i=1}^{4} g_{i}^{T}\left(h_{i}, \theta_{i}\right) D\left(\delta_{i}\right)\left[\begin{array}{c}
F_{x i} \\
F_{y i}
\end{array}\right] .
$$

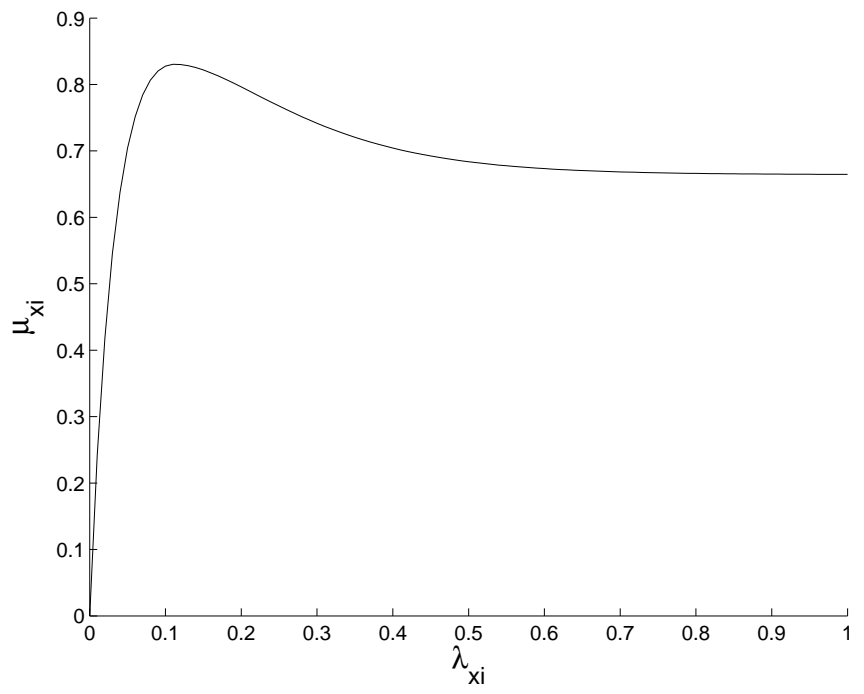

Figure 3: Longitudinal friction coefficient as a function of the longitudinal slip

Here,

$$
\begin{aligned}
T(v, \beta) & =\frac{1}{v}\left[\begin{array}{cc}
v \cos \beta & v \sin \beta \\
-\sin \beta & \cos \beta
\end{array}\right], v>0, \\
g_{i}\left(h_{i}, \theta_{i}\right) & =\left[\begin{array}{c}
-\sin \theta_{i} \\
\cos \theta_{i}
\end{array}\right] h_{i}, \\
D\left(\delta_{i}\right) & =\left[\begin{array}{cc}
\cos \delta_{i} & -\sin \delta_{i} \\
\sin \delta_{i} & \cos \delta_{i}
\end{array}\right] .
\end{aligned}
$$

Let the state vector be $x=\left[\begin{array}{lll}v & \beta & \dot{\psi}\end{array}\right]^{T}$ and the control input $u=\left[\begin{array}{llll}\lambda_{x 1} & \lambda_{x 2} & \lambda_{x 3} & \lambda_{x 4}\end{array}\right]^{T}$. We can then summarize the nonlinear state space model in the form

$$
\dot{x}=f\left(x, u, \delta_{W}, \mu_{H}\right) .
$$

We want to use feedback from the system state in the controller, and thus need measurements/estimates of this. The vehicle speed $v$ can be estimated from the wheel speeds and acceleration sensors, $\dot{\psi}$ can be measured and an observer can be designed to obtain the vehicle side slip angle $\beta$. We also need an estimate of the current friction coefficient $\mu_{H}$, which we assume is available from the ABS system (see e.g. $[5,10,11]$ ) or a separate system such as [7].

\section{Yaw Dynamics Control Problem Formulation}

The main goal of using brake actuators to control the yaw motion, is to be able to maintain steerability of a vehicle in critical driving situations in which the driver is unable to do this by using the steering wheel alone. We will use the same strategy of maintaining vehicle steerability as suggested in [10], which is by constraining the vehicle side slip angle $\beta$ and the yaw rate $\dot{\psi}$ to be within upper/lower bounds. We will in addition to this also place constraints on the control inputs. The authors of [10] addressed the state constraints indirectly by commanding 
the reference values of the states, using a linearized model and a pole placement approach. In our approach we will use the nonlinear dynamical model of the vehicle and an RHC optimal control strategy, to directly address these constraints. This strategy aims at computing an open loop optimal control trajectory on a time horizon. The first part of the control trajectory is applied to the is commanded to the vehicle's ABS system, and at the next sample the optimization is repeated, but now with a shifted horizon and a new initial condition from the new state. In this way this becomes a closed loop approach, see [12] for an overview.

The state and input constraints appear explicitly in the problem formulation as linear and nonlinear inequality constraints, while the state space model appears as nonlinear equality constraints. As the forces generated by the brakes are in negative $x$ direction, the control inputs $\lambda_{i}$ are constrained as

$$
\lambda_{i} \geq 0 \text { for } i \in\{1,2,3,4\} .
$$

The yaw rate $\dot{\psi}$ is constrained by [10]

$$
|\dot{\psi}| \leq \dot{\psi}_{\max }(v, \dot{v}, \beta)=\frac{a_{Y_{\max }}-\dot{v} \sin \beta}{v \cos \beta},
$$

where $a_{Y_{\max }}$ is the maximum lateral acceleration, given by

$$
a_{Y_{\max }}=\mu_{Y_{\max }} \cdot 8 \mathrm{~m} / \mathrm{s}^{2},
$$

and $\mu_{Y_{\max }}$ is the maximum lateral friction coefficient. Moreover, the side slip angle $\beta$ was in [10] constrained by a function of the velocity $v$, that is

$$
|\beta| \leq \beta_{\max }(v)=10^{\circ}-7^{\circ} \cdot \frac{v^{2}}{(40 \mathrm{~m} / \mathrm{s})^{2}} .
$$

Since our simulations indicated that (12) is generally satisfied when (10) is enforced, we chose to leave (12) implicit in the controller design to reduce controller complexity. These constraints are, however, shown in the simulations in Section 5. For simplicity, we consider a fixed $\mu_{h}=0.8$.

Let the horizon $[0, T]$ be divided into $N$ equal sized intervals by the ordered set of time instants $\left\{t_{1}, t_{2}, \ldots, t_{N}\right\} \subset[0, T]$. Let the input signal $u[0, T]$ be constant in each interval and parameterized by a vector $U=\left[\begin{array}{lll}u^{T}(0) & \cdots & u^{T}\left(t_{N-1}\right)\end{array}\right]^{T} \in$ $\mathbb{R}^{4 N}$, and let $X=\left[\begin{array}{lll}x^{T}\left(t_{1}\right) & \cdots & x^{T}\left(t_{N}\right)\end{array}\right]^{T} \in \mathbb{R}^{3 N}$ denote the vector of states at these time instants. By relaxing the inequality constraints to hold only at $N$ time instants, we can form the following dynamical optimal control problem (multiple shooting [4]):

$$
V(x(0))=\min _{U, X, s}\left(s^{T} W s+\int_{0}^{T} u(t)^{T} R u(t) d t\right)
$$

subject to the constraints,

$$
\begin{aligned}
\lambda_{x i}(t) & \geq 0, \quad i \in\{1,2,3,4\} \text { and } t \in\left\{t_{1}, \ldots, t_{N}\right\} \\
|\beta(t)| & \leq \beta_{\max }(v(t))+s_{\beta}, t \in\left\{t_{1}, \ldots, t_{N}\right\} \\
|\dot{\psi}(t)| & \leq \dot{\psi}_{\max }(v(t), \dot{v}(t), \beta(t))+s_{\psi}, t \in\left\{t_{1}, \ldots, t_{N}\right\} \\
x\left(t_{i+1}\right) & =\phi\left(x\left(t_{i}\right), u\left(t_{i}\right), t_{i+1}-t_{i}\right), i \in\{0, \ldots, N-1\}
\end{aligned}
$$

where $\phi(x(t), u(t), \tau)$ denotes the solution $x(t+\tau)$ to $\dot{x}=$ $f\left(x, u, \delta_{W}, \mu_{H}\right)$ with initial condition $x(t)$ and constant $u(t)$, $\delta_{W}$ and $\mu_{H}$ on the time interval between $t$ and $t+\tau$. Here, $s=$ $\left[\begin{array}{ll}s_{\beta} & s_{\dot{\psi}}\end{array}\right]$ are slack variables used to guarantee the existence of a feasible solution to the inequality constraints, the prediction horizon $T=0.6 s, N=3, R=I_{4 \times 4}$ and $W=2 I_{2 \times 2}$. The differential equations are solved by using the forward Euler method, with a discretization interval of $\Delta t=\frac{T \cdot N}{10}$. We assume during the prediction that the driver does not change the steering wheel angle. Note that this formulation does not assume any setpoints for the states. This means that as long as the inequality constraints are fulfilled on the horizon, the control input will be zero, so that the driver controls the vehicle by the steering angle alone. However, when the state constraints become active, the controller directs the appropriate forces to the individual brakes to keep the states within the constraints.

\section{Solution via mpNLP}

The optimization problem from the previous section is not well suited for a real-time application using an on-line NLP solver. This is mainly due to the high computational costs required to solve the NLP (13)-(17) at each sample. Instead, we obtain an approximate solution in the form of a piecewise linear (PWL) control law by using mpNLP [8]. The NLP (13)-(17) can be written in the general form,

$$
\begin{aligned}
\min _{z} J(z, \theta) & \\
G(z, \theta) & =0 \\
H(z, \theta) & \leq 0
\end{aligned}
$$

where $z^{T}=\left[\begin{array}{lll}U^{T} & X^{T} & s^{T}\end{array}\right]$ and $\theta^{T}=\left[\begin{array}{ll}x^{T} & \delta_{W}^{T}\end{array}\right]$. Eqs. (18)(20) are to be considered a multiparametric program, which means that $\theta$ is a parameter to the optimization problem, and the optimal solution is to be found for a range of parameter values. We seek to find an explicit approximate representation of the solution as a function of these parameters. The algorithm in [8] does this by partitioning the parameter space into a set of polyhedra, in which the NLP is approximated by a quadratic approximation. Each of these approximations can be treated as a multiparametric quadratic program (mpQP) which can be solved exactly off-line, giving the control input as an explicit PWL function of its time-varying parameters [3]. The method can briefly be summarized as follows (see [8] for details)

1) Let $\Theta$ be a polyhedral set of parameters $\theta$ in which the mpNLP is to be solved. Let $\Theta_{0}=\Theta$.

2) Select $\theta_{0}$ as the centre point of $\Theta_{0}$.

3) Solve the NLP (13)-(17) with $\theta=\theta_{0}$ by using an NLP solver to obtain the solution $U^{*}\left(\theta_{0}\right), X^{*}\left(\theta_{0}\right)$ and $s^{*}\left(\theta_{0}\right)$.

4) Compute a local quadratic approximation to the NLP cost function, and a linear approximation to the NLP constraints $G$ and $H$ at $\left(\theta_{0}, U^{*}\left(\theta_{0}\right), X^{*}\left(\theta_{0}\right), s^{*}\left(\theta_{0}\right)\right)$. Such an approximation defines an $\mathrm{mpQP}$. 
5) Estimate the maximal errors (in cost function, control inputs and constraint violations, see [8] for details) made by approximating the mpNLP solution with the mpQP solution for $\theta \in \Theta_{0}$. If these are not within apriori defined bounds, sub-partition $\Theta_{0}$ into two polyhedral regions.

6) Select a new $\Theta_{0}$ from the partition. If no further subpartitioning is needed, go to step 7. Otherwise, go to Step 2.

7) For every sub-partition $\Theta_{0}$, solve the local mpQP problem.

This means that the main effort of solving the optimization problem is moved off-line. Efficient off-line mpQP solvers have recently been developed [14]. The on-line effort is reduced to evaluating a PWL function, which can be implemented efficiently using a binary search tree as in [15].

The explicit solution obtained in this paper was computed for

$$
\left[\begin{array}{c}
75 \frac{\mathrm{km}}{h} \\
-7^{\circ} \\
-20^{\circ} / \mathrm{s} \\
-7^{\circ}
\end{array}\right] \leq\left[\begin{array}{c}
v \\
\beta \\
\dot{\psi} \\
\delta_{W}
\end{array}\right] \leq\left[\begin{array}{c}
125 \frac{\mathrm{km}}{\mathrm{h}} \\
2.5^{\circ} \\
20^{\circ} / \mathrm{s} \\
7^{\circ}
\end{array}\right]
$$

We assume that $\beta$ cannot have a too large positive value for a positive $\dot{\psi}$. This assumption was made to decrease the complexity of the controller, this may, however, need to be reconsidered in a real vehicle. The range of speeds would also have to be increased.

The whole solution vector $z$ of the explicit solution does not need to be evaluated, as $u(t)$ is the only part needed in the control law. The explicit solution obtained consists of 5803 regions in the 4-dimensional parameter space, and the binary search tree representing the control law can be stored using 23909 numbers, needing a maximum of 173 arithmetic operations per sample to evaluate the control law. The maximum allowed estimated error in the control input $\left(\lambda_{x i}\right)$ was specified to 0.04 , while an estimated error of $25 \%$ was allowed in the cost function. The actual errors are, however, typically orders of magnitude smaller. Note that as the NLP is non-convex, care must be taken to ensure that the global optimizer is obtained. This is another argument for using an explicit solution approach, as this gives the possibility of off-line verification of the solution. The nonlinear optimization problems were solved by NPSOL [6].

\section{Simulations}

We have considered a simulation example, in which the driver must carry out an evasive maneuver at high speed, with $\mu_{H}=$ 0.8 , corresponding to a dry asphalt surface. The driver controls the steering angle, and the controller applies an appropriate distribution of braking forces to keep the state within the constraints. Figure 4 shows the uncontrolled behavior of the system. The vehicle is obviously unable to follow the drivers command. The yaw rate increases in the left turn, but the vehicle does not respond when the driver makes the right turn. The dotted lines in the $\beta$ - and $\dot{\psi}$-plots are the constraints (15)-(16).
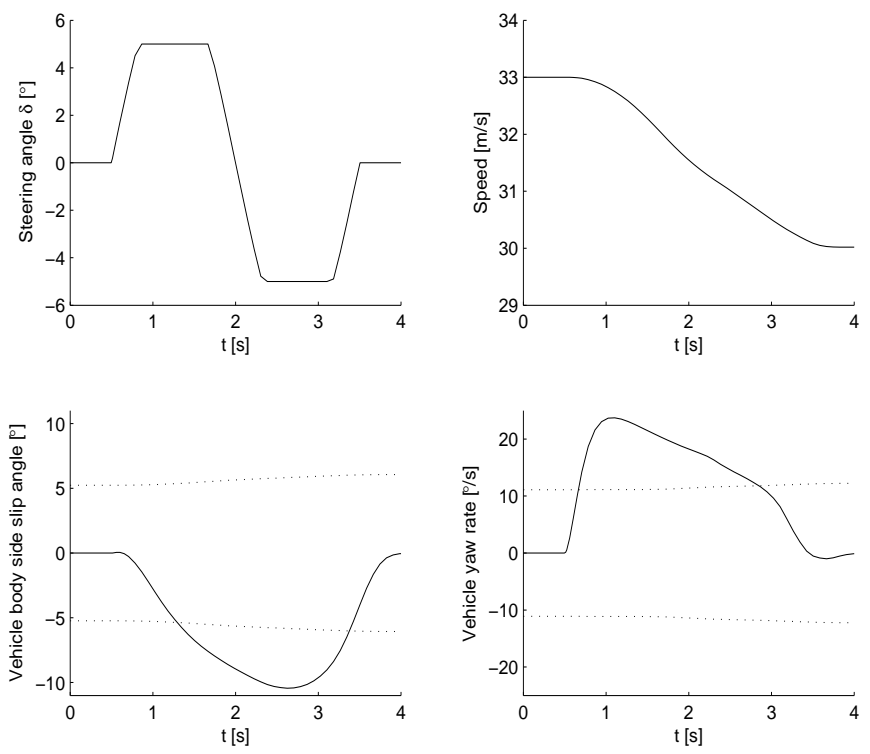

Figure 4: Simulation of vehicle with no control action
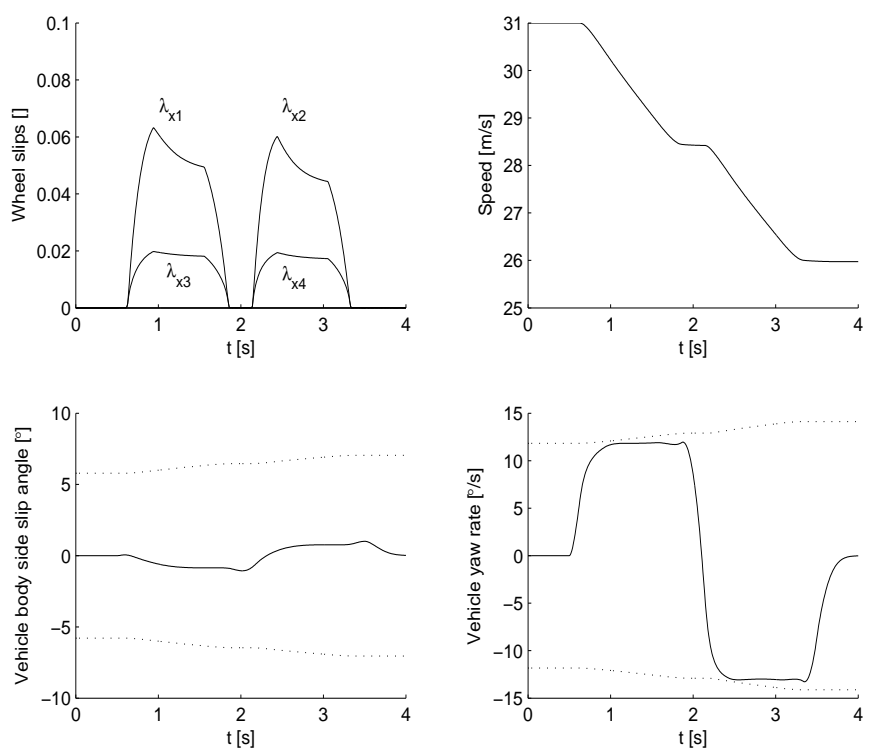

Figure 5: Simulation with optimal control obtained from NLP solution

Figure 5 shows the same maneuver, but now with optimal control applied, obtained by using the exact NLP solution. In the first turn the controller applies a braking force to both right wheels to keep the yaw rate within its bounds. A production ESP controller would in such situations apply a force to the front wheel only. This is due to the fact that $F_{y i}$ decreases when a wheel is braking (see Figure 2), and one wants to keep the side force on the back wheels. However, the optimal (in the sense of the previously defined optimization problem) control 

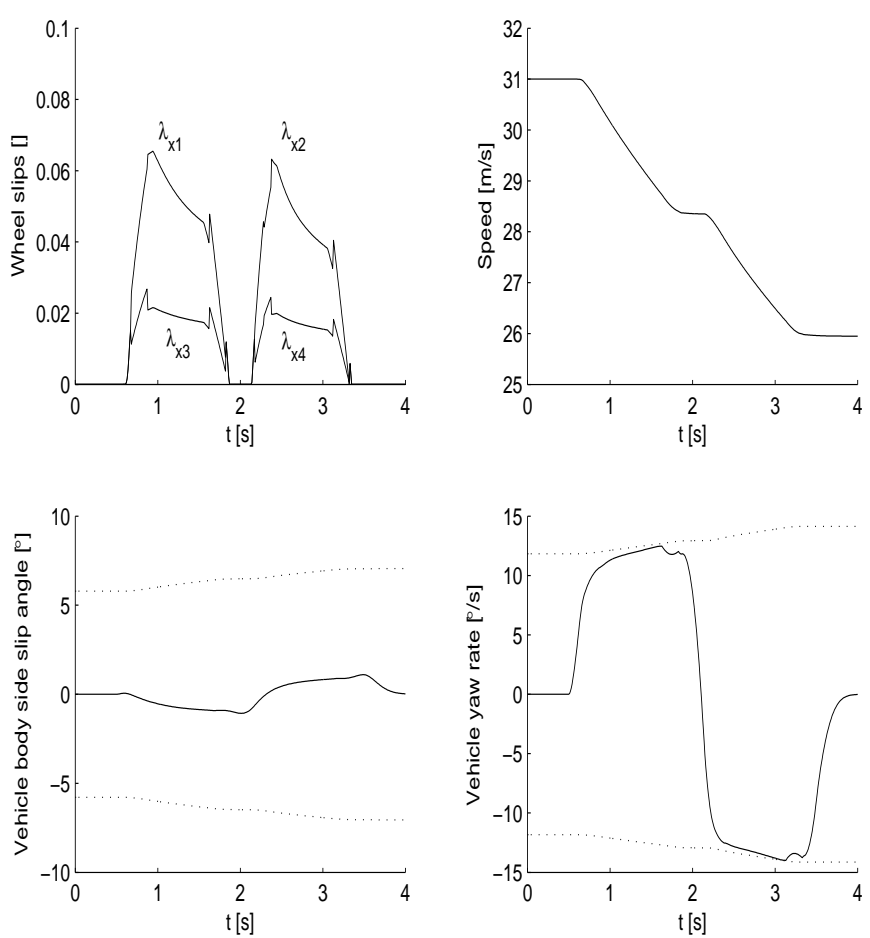

Figure 6: Simulation with sub-optimal explicit PWL mpNLP solution
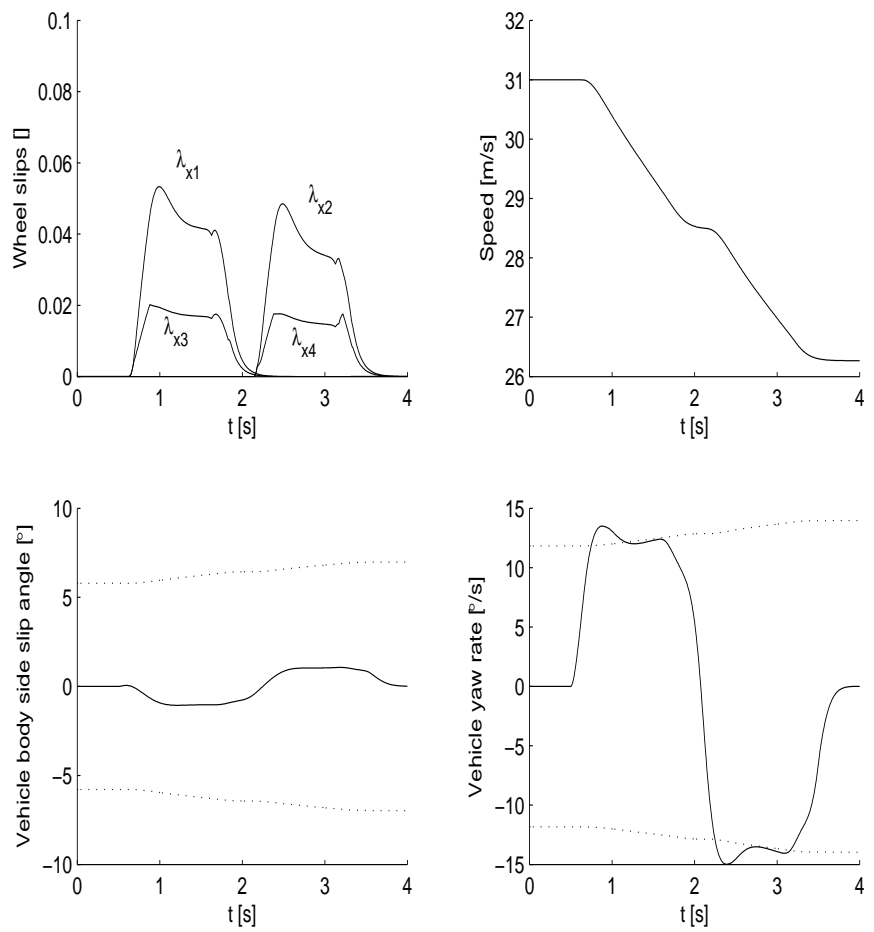

Figure 7: Simulation with unmodelled actuator dynamics
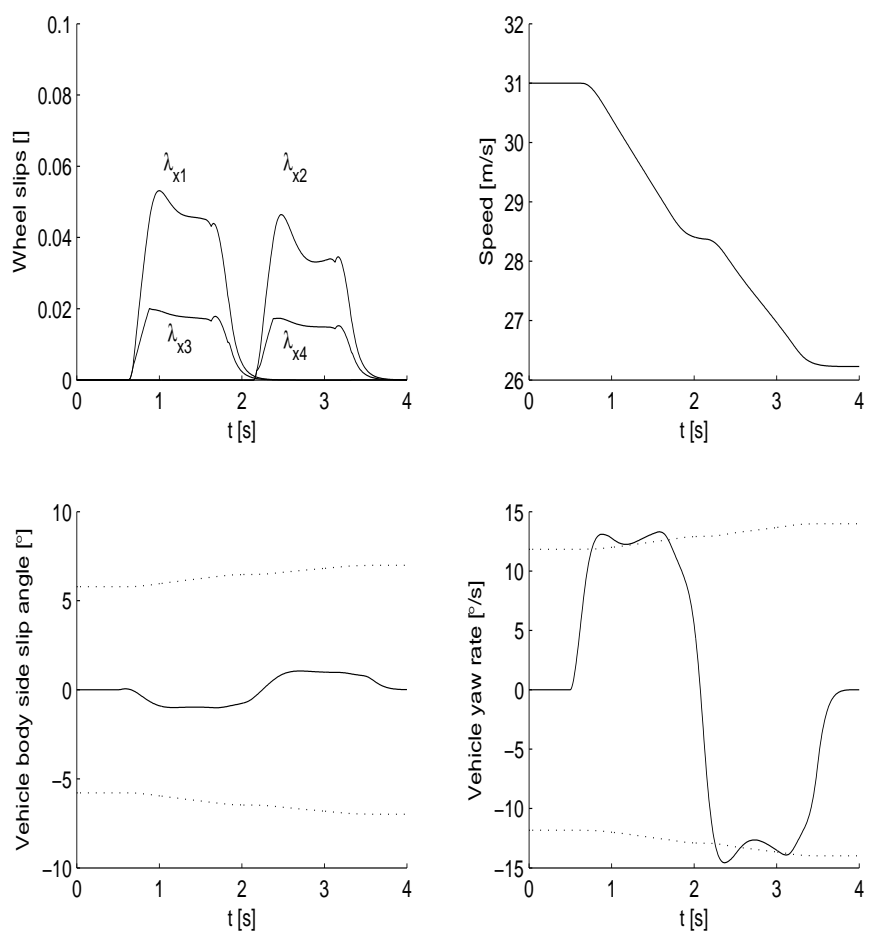

Figure 8: Simulation with unmodelled actuator dynamics and varying $\mu_{H}(t)=0.8+0.08 \cdot \sin (\pi t)$
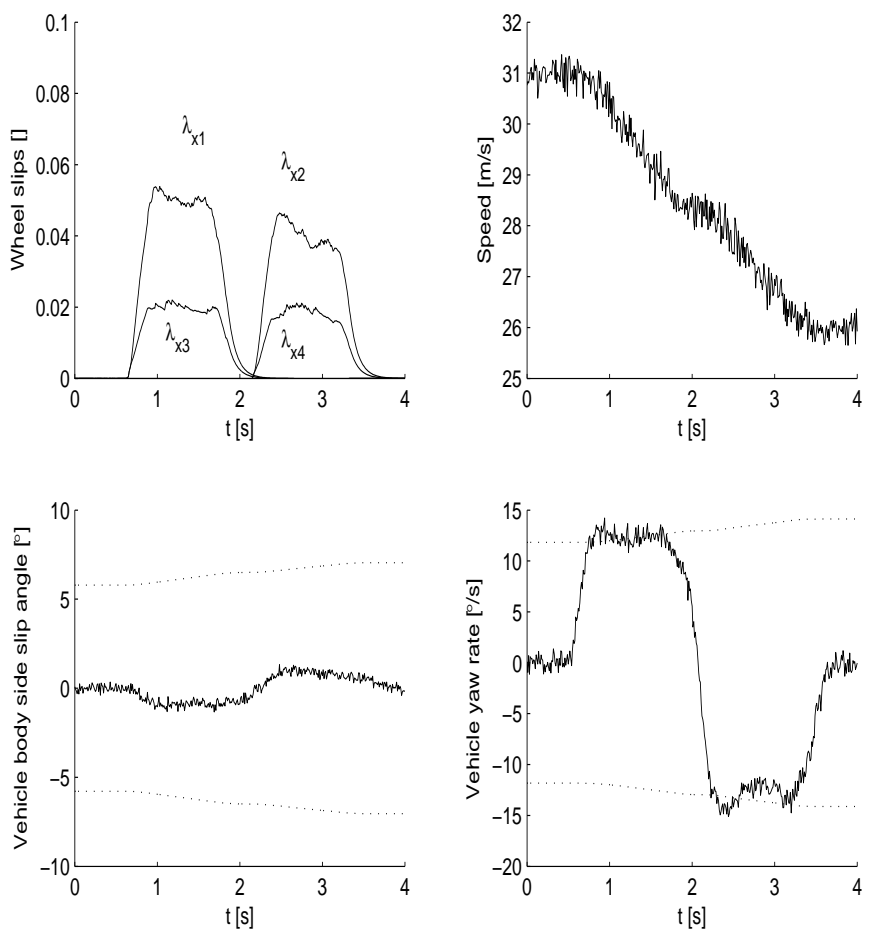

Figure 9: Simulation with unmodelled actuator dynamics, varying $\mu_{H}$ and measurement noise (the curves show the states with noise included) 
action is to apply a small force to the rear wheel also. Since only a small slip is requested on the rear wheels, most of the lateral force $F_{y i}$ is retained, while the longitudinal force $F_{x i}$ contributes to keeping control of the yaw dynamics. In the second turn, a similar braking torque is applied to the left wheels.

In Figure 6, the explicit PWL control law described in Section 4 is applied. The control action approximates the optimal one, and only minor discrepancies can be seen in the state trajectory. The control input is however discontinuous at some time instants. The discontinuities occur when switching from one quadratic approximation to another. Even if one would prefer a continuous control input, the effects of the small discontinuity are not prohibitive. The obtained control inputs are used as setpoints for slip controllers on each wheel, and the slip actuator dynamics and ABS system work as a low-pass filter. In Figure 7 this effect is illustrated by including unmodelled actuator dynamics, which are approximated as

$$
\frac{\lambda_{x i}}{\lambda_{x i}^{*}}(s)=\frac{1}{1+0.1 s}
$$

where $\lambda_{x i}^{*}$ is the setpoint of the slip controller. This gives a small overshoot in the yaw rate. The actuator dynamics are not accounted for in the controller design. The estimation of the maximum friction coefficient $\mu_{H}$ would include some error, and Figure 8 also includes the effect of varying $\mu_{H}$ within $\pm 10 \%$ of its nominal value. In Figure 9 measurement noise is also added. We conclude that the control strategy appears to be robust and performs well.

\section{Conclusions}

We have presented an optimization based constrained controller for automotive vehicle lateral stabilization. Simulations have been made on a case in which the vehicle loses steerability under manual control, but with the controller applied, the maneuver remains stable. The proposed solution may be well suited for implementation in a real vehicle, as it is computationally very efficient. A practical implementation may, however, require some extensions: a) The controller should be designed to work for all values of $\mu_{H}$. b) The simplification that the vehicle mass is equally distributed among the four wheels may be improved. c) The range of parameter values for which the control law is designed should be increased. d) Interactions with the ABS system and observer design may require further attention. At least points a) and c) above will lead to increased complexity in terms of a larger number of regions in the representation of the PWL controller. This may be unacceptable, but may be counteracted in several ways:

- Tuning of approximation tolerances

- Approximating and joining solution in neighboring small regions

- Separating the control problem into a control allocation problem (see [9]) and a simpler control problem. Such a control allocation problem can be posed as an mpNLP, which would be simpler than the mpNLP obtained from the RHC formulation in this paper.

\section{Acknowledgements}

This work was financed by the Norwegian Research Council, project no. 134553/410. The authors would like to thank Dr. Jens Kalkkuhl, DaimlerChrysler for valuable comments.

\section{References}

[1] J. Ackermann, J. Guldner, W. Sienel, R. Steinhauser, and V.I. Utkin. Linear and nonlinear controller design for robust automatic steering. IEEE Transactions on Control Systems Technology, 3(1):132-143, 1995.

[2] H. Bauer, editor. ESP Electronic Stability Program. Robert Bosch GmbH, Stuttgart, first edition, 1999.

[3] A. Bemporad, M. Morari, V. Dua, and E. N. Pistikopoulos. The explicit linear quadratic regulator for constrained systems. Automatica, 38(1):3-20, 2002.

[4] H. G. Bock, M. M. Diehl, D. B. Leineweber, and J. P. Schlöder. A direct multiple shooting method for real-time optimization of nonlinear DAE processes. In Nonlinear model predictive control (Ascona, 1998), pages 245-267. Birkhäuser, Basel, 2000.

[5] M. Burckhardt. Fahrwerktechnik: Radschlupf-Regelsysteme. Vogel Verlag, Würzburg, 1993.

[6] P. E. Gill, W. Murray, M. A. Saunders, and M. H. Wright. Users guide for NPSOL 5.0: A fortran package for nonlinear programming. Technical Report SOL 86-2, Systems Optimization Laboratory, Department of Operations Research, Stanford University, 1998.

[7] F. Gustafsson. Slip-based tire-road friction estimation. Automatica, 33:1087-1099, 1997.

[8] T. A. Johansen. On multi-parametric nonlinear programming and explicit nonlinear model predictive control. In Proc. 41 st IEEE Conf. on Decision and Control, volume 3, pages 27682773, Las Vegas, NV, 2002.

[9] T. A. Johansen, T. I. Fossen, and P. Tøndel. Efficient optimal constrained control allocation via multi-parametric programming. J. Guidance, Control and Dynamics, 2003. Submitted.

[10] U. Kiencke and L. Nielsen. Automotive Control Systems. Springer-Verlag, 2000.

[11] Jens Lüdemann. Heterogeneous and Hybrid Control with Application in Automotive Systems. PhD thesis, Glasgow University, 2002.

[12] D. Q. Mayne, J. B. Rawlings, C. V. Rao, and P. O. M. Scokaert. Constrained model predictive control: Stability and optimality. Automatica, 36(6):789-814, 2000.

[13] I. Petersen, T. A. Johansen, J. Kalkkuhl, and J. Lüdemann. Wheel slip control in ABS brakes using gain scheduled constrained LQR. In Proc. European Contr. Conf., Porto, 2001.

[14] P. Tøndel, T. A. Johansen, and A. Bemporad. An algorithm for multi-parametric quadratic programming and explicit MPC solutions. Automatica, 39(3):489-497, 2003.

[15] P. Tøndel, T. A. Johansen, and A. Bemporad. Computation of piecewise affine control via binary search tree. Automatica, 39(5):945-950, 2003. 